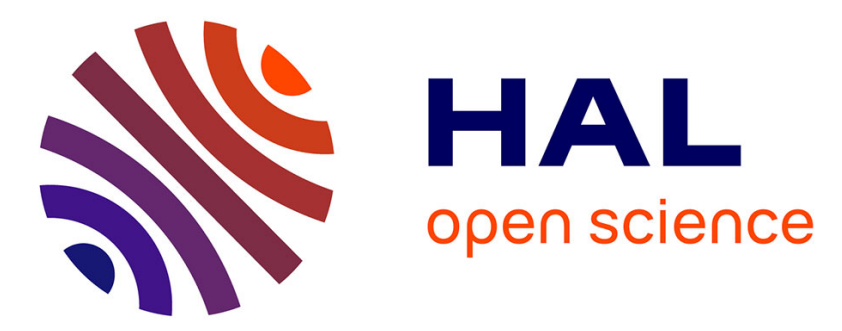

\title{
Reshaping the physical properties of a quadrotor through IDA-PBC and its application to aerial physical interaction
}

\author{
Burak Yüksel, Cristian Secchi, Heinrich H Bülthoff, Antonio Franchi
}

\section{- To cite this version:}

Burak Yüksel, Cristian Secchi, Heinrich H Bülthoff, Antonio Franchi. Reshaping the physical properties of a quadrotor through IDA-PBC and its application to aerial physical interaction. 2014 IEEE Int. Conf. on Robotics and Automation, May 2014, Hong Kong, China. 8p., 10.1109/ICRA.2014.6907782 . hal-01137967

\author{
HAL Id: hal-01137967 \\ https://hal.science/hal-01137967
}

Submitted on 31 Mar 2015

HAL is a multi-disciplinary open access archive for the deposit and dissemination of scientific research documents, whether they are published or not. The documents may come from teaching and research institutions in France or abroad, or from public or private research centers.
L'archive ouverte pluridisciplinaire HAL, est destinée au dépôt et à la diffusion de documents scientifiques de niveau recherche, publiés ou non, émanant des établissements d'enseignement et de recherche français ou étrangers, des laboratoires publics ou privés. 


\title{
Reshaping the Physical Properties of a Quadrotor through IDA-PBC and its Application to Aerial Physical Interaction
}

\author{
Burak Yüksel $^{1}$, Cristian Secchi ${ }^{2}$, Heinrich H. Bülthoff ${ }^{1}$ and Antonio Franchi ${ }^{3,1}$
}

\begin{abstract}
In this paper we propose a controller, based on an extension of Interconnection and Damping AssignmentPassivity Based Control (IDA-PBC) framework, for shaping the whole physical characteristics of a quadrotor and for obtaining a desired interactive behavior between the robot and the environment. In the control design, we shape the total energy (kinetic and potential) of the undamped original system by first excluding external effects. In this way we can assign a new dynamics to the system. Then we apply damping injection to the new system for achieving a desired damped behavior. Then we show how to connect a high-level control input to such system by taking advantage of the new desired physics. We support the theory with extensive simulations by changing the overall behavior of the UAV for different desired dynamics, and show the advantage of this method for sliding on a surface tasks, such as ceiling painting, cleaning or surface inspection.
\end{abstract}

\section{INTRODUCTION}

Multi-rotor UAVs used as flying robotic systems have been very popular research tools for the last decade especially in the sense of developing new control techniques. The usually simple mechanics of these platforms, like the quadrotor UAV, allow to develop advanced controllers, while dexterity of their workspace makes them important for observation and manipulation tasks. Most of the works have considered the system as a flying sensor, and developed controllers for trajectory tracking [1], [2], haptic teleoperation [3], [4], robot vision [5], and distributed control [6], [7].

The direction of recent studies is leading the scientists to the field of aerial manipulation, where these flying UAVs are no longer just passive observers, but flying robots physically interacting with their environment [8], [9]. This interaction could be achieved by the quadrotor itself [10], [11]; or by using some manipulation tools such as cables [12], a manipulator arm [13], [14], [15], a rigid [16], [17], or a flexible link [18]. Different control techniques are applied to these different designs. The controller in [10] used Kalman filters to estimate external forces from position and attitude information. The controller is proposed for linearized translational dynamics in near-hovering case, which provides a local solution in terms of physical interaction for a quadrotor. In [11] a hybrid pose and wrench control framework is used for stable contact of quadrotors, where the wrench is estimated using pose measurements and control inputs

\footnotetext{
${ }^{1}$ Max Planck Institute for Biological Cybernetics, Spemanstr. 38, 72076, Tübingen, Germany. \{burak. yueksel, hhb\}@tuebingen.mpg. de

${ }^{2}$ DISMI-University of Modena and Reggio Emilia, Via G. Amendola 2, Morselli Building, 42122 Reggio Emilia Italy. cristian.secchi@unimore.it

${ }^{3}$ Centre National de la Recherche Scientifique (CNRS), Laboratoire d'Analyse et d'Architecture des Systèmes (LAAS), 7 Avenue du Colonel Roche, 31077 Toulouse CEDEX 4, France. antonio.franchillaas.fr
}

by the help of PI(D) controllers. In order to robustly deal with poorly structured environment, impedance control has been exploited. In [13] a Cartesian impedance control for regulating the stiffness and the damping of a manipulator mounted on an UAV has been proposed. The approaches presented in [19] and [14] exploit passivity based control for shaping only the potential energy of a quadrotor and for setting a desired cartesian stiffness to the controlled system. Potential energy is only one of the factors affecting the way a mechanical system interacts with the environment. Inertial properties and damping also play a major role for determining the interactive behavior. Furthermore, since the direction of the thrust of a quadrotor depends on the orientation of the system, it is not sufficient to shape the Cartesian impedance for achieving an effective control of interaction.

The main motivation of this paper is to control a quadrotor for shaping its whole physical behavior by changing its inertia, the potential field it is immersed in and its damping, both for Cartesian and orientation components. In other words, we would like to transform a quadrotor in another physical system that is more suitable for a given objective. In this way we can control the whole interactive behavior of the quadrotor and we can increase the accuracy of the interactive task, e.g., reducing the oscillations in the case of contact with a quickly moving object, being more compliant when the contact surface has a variable profile or being lighter when approaching a fragile environment.

In order to attain this goal, we recast the problem of interaction control for a quadrotor in the port-Hamiltonian framework. We build a port-Hamiltonian model of a properly precompensated dynamics of the quadrotor and we exploit and extend the Interconnection and Damping Assignment Passivity Based Control (IDA-PBC) framework [20], [21] for shaping the total energy of the system. Furthermore, we propose a damping injection and scaling technique for setting the desired damping and for achieving the desired controlled dynamics.

The paper is organized as follows. Section II gives a background on port-Hamiltonian systems and control design using IDA-PBC. In Sec. III we rewrite the full dynamics of a precompensated quadrotor in a port-Hamiltonian form and in Sec. IV we introduce the controller for shaping the physics of the quadrotor. Simulation results are given in Sec. V, to support the proposed method, where we show how the behavior of overall system can be changed using the proposed strategy and how this can be exploited in an application case where the quadcopter needs to slide a tool over an uneven surface. Finally Sec. VI concludes the paper with useful remarks and ideas for future work. 


\section{BACKGROUND}

In this section we will provide some background on port-Hamiltonian systems and on IDA-PBC control. More information can be found in [22], [20] and [21].

The port-Hamiltonian framework is a generalization of standard Hamiltonian mechanics and energetic features play a primary role in the modeling process. The most common representation of a port-Hamiltonian system is the following:

$$
\left\{\begin{array}{l}
\dot{x}=[\mathcal{J}(x)-\overline{\mathcal{R}}(x)] \frac{\partial H}{\partial x}+G(x) u \\
y=G(x)^{T} \frac{\partial H}{\partial x}
\end{array}\right.
$$

where $x \in \mathbb{R}^{n}$ is the state and $H(x): \mathbb{R}^{n} \rightarrow \mathbb{R}$ represents the amount of energy stored in the system. Matrices $\mathcal{J}(x)=$ $-\mathcal{J}(x)^{T}$ and $\overline{\mathcal{R}}(x) \geq 0$ represent the internal energetic interconnections and the dissipation of the port-Hamiltonian system, respectively. Furthermore, $G(x)$ is the input matrix and the input-output pair $(u, y)$ represents a power port, namely a pair of variables whose product gives (generalized) power that is either stored or dissipated by the system.

Using IDA-PBC [20] and its extension proposed in [21] it is possible to control a port-Hamiltonian system in such a way that it behaves as a target dynamics, namely as a new port-Hamiltonian system with a desired interconnection matrix, damping matrix and energy function and even with a different state variable $\bar{x} \in \mathbb{R}^{n}$. Formally, let

$$
x=\Phi(\bar{x}, t)
$$

be the map relating $\bar{x}$ and $x$, where $\Phi$ and $\frac{\partial \Phi}{\partial \bar{x}}$ are invertible at any time $t$. Let $\mathcal{J}_{d}, \mathcal{R}_{d}$ and $H_{d}$ be the desired interconnection matrix, dissipation matrix and energy function, respectively. The port-Hamiltonian system in (1) can be transformed into the target port-Hamiltonian dynamics described by

$$
\dot{\bar{x}}=\left[\mathcal{J}_{d}(\bar{x})-\overline{\mathcal{R}}_{d}(\bar{x})\right] \frac{\partial H_{d}}{\partial \bar{x}}
$$

using

$$
\begin{aligned}
& u=\left(G^{T}(x) G(x)\right)^{-1} G^{T}(x)\left[\frac{\partial \Phi}{\partial \bar{x}}\left(\mathcal{J}_{d}(\bar{x})-\overline{\mathcal{R}}_{d}(\bar{x})\right) \frac{\partial H_{d}}{\partial \bar{x}}-\right. \\
& \left.-(\mathcal{J}(x)-\overline{\mathcal{R}}(x)) \frac{\partial H}{\partial x}+\frac{\partial \Phi}{\partial t}\right]
\end{aligned}
$$

where $\left(G^{T}(x) G(x)\right)^{-1} G^{T}(x)$ is the pseudoinverse of $G(x)$, if and only if the following matching equation holds:

$$
\begin{aligned}
G^{\perp}(x)\left[\frac{\partial \Phi}{\partial \bar{x}}\left(\mathcal{J}_{d}(\bar{x})-\overline{\mathcal{R}}_{d}(\bar{x})\right) \frac{\partial H_{d}}{\partial \bar{x}}+\frac{\partial \Phi}{\partial t}-\right. \\
\left.-(\mathcal{J}(x)-\overline{\mathcal{R}}(x)) \frac{\partial H}{\partial x}\right]=0
\end{aligned}
$$

where $G^{\perp}(x)$ is the full rank left annihilator of $G(x)$.

The main drawback of IDA-PBC is the necessity of solving the nonlinear partial differential equations (PDE) (5). In general it is not possible to find a closed form solution of the matching equation and, therefore, it is not possible to find all the possible achievable target dynamics. In practice, it is necessary to test if the desired target dynamics is achievable and, if not, to modify it until (5) is satisfied.

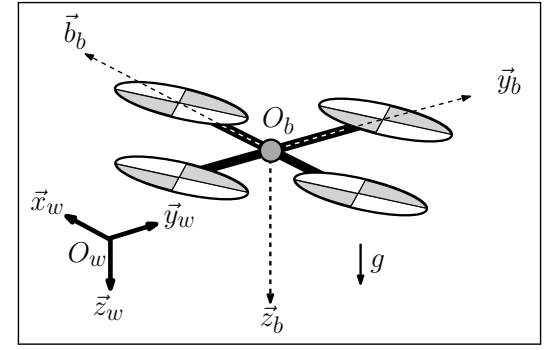

Fig. 1. Schematic figure of a quadrotor and notation used in the paper.

\section{PORT-HAMILTONIAN MODEL OF A QUADROTOR}

Consider an inertial world frame $\mathcal{W}:\left\{O_{w}, \vec{x}_{w}, \vec{y}_{w}, \vec{z}_{w}\right\}$ and a body frame $\mathcal{B}:\left\{O_{b}, \vec{b}_{b}, \vec{y}_{b}, \vec{z}_{b}\right\}$ rigidly attached to the quadrotor and with the origin placed in the quadrotor center of mass. Both frames follow the NED (North-East-Down) convention. The dynamic equation of the quadrotor system shown in Fig. 1 is given by in two parts [3]: rotational

$$
\Sigma_{1}: \begin{cases}J \dot{\omega} & =-S(\omega) J \omega+\bar{\tau}+\tau_{e} \\ \dot{\eta} & =T(\eta) \omega\end{cases}
$$

and the translational

$$
\Sigma_{2}: \quad\left\{m \ddot{x}_{b}=-\rho R(\eta) e_{3}+m g e_{3}+f_{e}\right.
$$

where $m>0$ is the mass of the quadrotor; $e_{3}=\left(\begin{array}{lll}0 & 0 & 1\end{array}\right)^{T}$; $x_{b} \in \mathbb{R}^{3}$ is the position of $O_{b}$ in $\mathcal{W} ; \eta=[\phi \theta \psi]^{T} \in \mathbb{R}^{3}$ are the roll, pitch, and yaw angles representing the orientation of $\mathcal{B}$ in $\mathcal{W} ; R(\eta) \in S O(3)$ is the associated rotational matrix; $\rho \in \mathbb{R}$ is the thrust ${ }^{1}$ control input along $-\vec{z}_{b} ; \omega \in \mathbb{R}^{3}$ is the angular velocity of $\mathcal{B}$ w.r.t. to $\mathcal{W}$ expressed in $\mathcal{B} ; J \in$ $\mathbb{R}^{3 \times 3}$ is the inertia matrix w.r.t. the body frame; $g$ is the gravity acceleration directed along $\vec{z}_{w} ; \bar{\tau} \in \mathbb{R}^{3}$ and $\tau_{e} \in$ $\mathbb{R}^{3}$ represent the torque input and the external (environment) torque, respectively, both expressed in $\mathcal{B} ; f_{e} \in \mathbb{R}^{3}$ is the external (environment) force expressed in $\mathcal{W} ; T(\eta) \in \mathbb{R}^{3}$ is the matrix relating $\omega$ with $\dot{\eta}$; and $S(\star): \mathbb{R}^{3} \rightarrow s o(3)$ is the skew-symmetric operator.

In order to simplify the structure of the matching condition and, consequently, to enlarge the set of target dynamics that can be achieved, we consider a control input $\bar{\tau}$ similar to [3] but without near-hovering purposes, defined as

$$
\bar{\tau}=J T^{-1}\left[\left(-k_{d}+Q\right) \dot{\eta}+\tau+\left(I-T J^{-1}\right) \tau_{e}\right]
$$

where $k_{d} \in \mathbb{R}^{+}$and $\tau=\left[\begin{array}{lll}\tau_{1} & \tau_{2} & \tau_{3}\end{array}\right]^{T} \in \mathbb{R}^{3}$ and

$$
Q=T \dot{T}^{-1}+T J^{-1} S(\omega) J T^{-1}
$$

Therefore, the rotational dynamics in (6) can be rewritten by applying (8) as follows

$$
\ddot{\eta}=-k_{d} \dot{\eta}+\tau+\tau_{e}
$$

${ }^{1}$ In normal situations it is actually $\rho>0$, see, e.g., the simulations of Sec. V. However, if needed by the task, negative thrust can always be achieved in the implementation, as, e.g., in [23]. 
where $I$ is the identity matrix of proper dimension. The plant represented by (7) and (10) can be modeled as a mechanical port-Hamiltonian system. Let $M \in \mathbb{R}^{6 \times 6}$ be

$$
M=\left(\begin{array}{cc}
m I & 0 \\
0 & I
\end{array}\right)
$$

where 0 is the zero matrix of proper dimension. Let $q=$ $\left(x_{b}^{T} \eta^{T}\right)^{T}=\left\{q_{1}, \cdots, q_{6}\right\} \in \mathbb{R}^{6}$ and $p=M \dot{q} \in \mathbb{R}^{6}$ be the configuration and momentum variables. Furthermore, let $u=$ $\left(\rho, \tau^{T}\right)^{T} \in \mathbb{R}^{4}$ be the input vector. The quadrotor dynamics can be rewritten as:

$$
\left(\begin{array}{l}
\dot{q} \\
\dot{p}
\end{array}\right)=\left[\left(\begin{array}{cc}
0 & I \\
-I & 0
\end{array}\right)-\left(\begin{array}{cc}
0 & 0 \\
0 & \mathcal{R}
\end{array}\right)\right]\left(\begin{array}{l}
\frac{\partial H}{\partial q} \\
\frac{\partial H}{\partial p}
\end{array}\right)+\left(\begin{array}{cc}
0 & 0 \\
G & I
\end{array}\right)\left(\begin{array}{c}
u \\
w_{e}
\end{array}\right)
$$

where $\mathcal{R}=k_{d} I$ models the dissipation introduced by (8) and $w_{e}=\left(f_{e}^{T}, \tau_{e}^{T}\right)^{T}$ represents the external wrench acting on the quadrotor. The total energy function and the input submatrix $G$ are given by:

$$
\begin{aligned}
& H(q, p)=\frac{1}{2} p^{T} M^{-1} p+V(q)=\frac{1}{2} p^{T} M^{-1} p-m g q_{3} \\
& G=\left(\begin{array}{cc}
G_{1} & 0 \\
0 & I
\end{array}\right) \in \mathbb{R}^{6 \times 4} \quad \text { with } \quad G_{1}=-R e_{3} \in \mathbb{R}^{3}
\end{aligned}
$$

It can be shown that the quadrotor has the property of cyclo-passivity [24], namely it cannot create energy over closed paths in the state space. Passivity, a stronger property, cannot be proven because the gravitational potential energy $V(q)$, and, consequently, the total energy (13) is not lower bounded.

Proposition 1: The system (12) is cyclo-passive with respect to the pair

$$
\left(\left(\begin{array}{c}
u \\
w_{e}
\end{array}\right),\left(\begin{array}{c}
G^{T} \frac{\partial H}{\partial p} \\
\frac{\partial H}{\partial p}
\end{array}\right)\right)
$$

Proof: Consider the energy function defined in (13). Using (12) we obtain:

$$
\begin{aligned}
\dot{H} & =\left(\begin{array}{ll}
\frac{\partial^{T} H}{\partial q} & \frac{\partial^{T} H}{\partial p}
\end{array}\right)\left(\begin{array}{l}
\dot{q} \\
\dot{p}
\end{array}\right) \\
& =-\frac{\partial^{T} H}{\partial p} \mathcal{R} \frac{\partial H}{\partial p}+\frac{\partial^{T} H}{\partial p} G u+\frac{\partial^{T} H}{\partial p} w_{e}
\end{aligned}
$$

Considerng that $\mathcal{R} \geq 0$ we obtain that

$$
\dot{H} \leq \frac{\partial^{T} H}{\partial p} G u+\frac{\partial^{T} H}{\partial p} w_{e}
$$

which proves the statement.

Remark 1: The cyclopassivity property can be interpreted as an extension of the more standard passivity property. It requires that the system behaves as a physical system from an energetic point of view (i.e., that the energy introduced into the system from the external world is either stored or dissipated) but it does not require that the energy function is lower bounded. Cyclopassivity, unlike passivity, prevents from proving the stability of an equilibrium point of the unforced system but, nevertheless, this is consistent with the physics of the quadrotor that has no equilibrium points in case all the inputs (both the control input and the external wrench) are null.

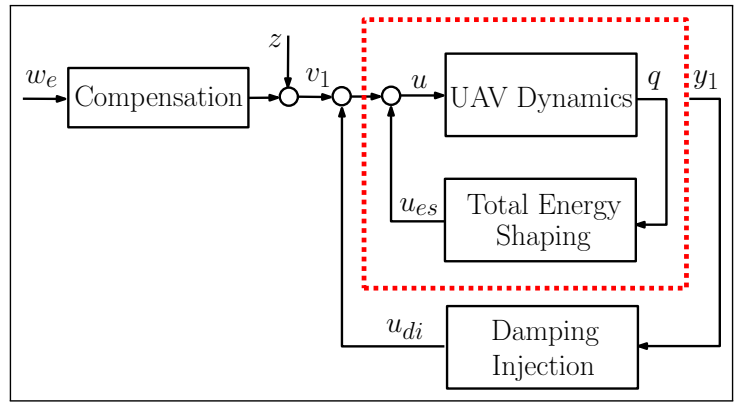

Fig. 2. Control Design using IDA-PBC and Damping Injection.

\section{CONTROLLER DESIGN}

In this section we will exploit and extend the IDAPBC formulation presented in [21] in order to completely change the physical properties of the quadrotor and the way it reacts to external forces and torques. In other words, rather than controlling the position or the velocity, we aim at transforming the quadrotor into a physically different quadrotor that reacts as a new desired physical system to external solicitations.

More formally, we aim at controlling (12) in such a way that it behaves as a new mechanical system described by:

$$
\left(\begin{array}{c}
\dot{q} \\
\dot{p}
\end{array}\right)=\left[\left(\begin{array}{cc}
0 & I \\
-I & 0
\end{array}\right)-\left(\begin{array}{cc}
0 & 0 \\
0 & \mathcal{R}_{d}
\end{array}\right)\right]\left(\begin{array}{c}
\frac{\partial H_{d}}{\partial q} \\
\frac{\partial H_{d}}{\partial \bar{p}}
\end{array}\right)+\left(\begin{array}{l}
0 \\
I
\end{array}\right) \tilde{w}_{e}
$$

where the new state $\bar{p}=M_{d} \dot{q}$ is the new momentum, associated with the new inertia matrix $M_{d}$ that is chosen to be constant and with the following structure:

$$
M_{d}=\left(\begin{array}{cc}
m_{d} I & 0 \\
0 & N
\end{array}\right)
$$

where $m_{d} \in \mathbb{R}^{+}$and $N \in \mathbb{R}^{3 \times 3}$ is a symmetric positive definite matrix representing the desired mass and the desired rotational inertia respectively. The desired energy function is

$$
H_{d}=\frac{1}{2} \bar{p}^{T} M_{d}^{-1} \bar{p}+V_{d}(q) .
$$

The choice of $M_{d}$ has been made in order to mimic the structure of (11) such that the controlled system will have an inertia that is consistent with the mechanics of the quadrotor. Furthermore, (18) has the advantage of decoupling rotational and Cartesian kinetic energy simplifying the design of the IDA-PBC control law. The desired potential function $V_{d}$ can be any function such that the matching equation of the IDAPBC is satisfied. $\mathcal{R}_{d}$ is the desired dissipation matrix that will also be constrained by the underactuation of the quadrotor. Finally, $\tilde{w}_{e}$ is the partially compensated external wrench and it will be defined more clearly later in this section.

The control law, whose block diagram is depicted in Fig. 2, will be designed in two steps. In the first step (developed in Sec. IV-A) the non conservative wrenches will be disregarded and the internal energetic structure of the quadrotor will be shaped. In the second step (detailed in Sec. IV-B) dissipation and external wrench will be considered and the control input will be adjusted in such a way to achieve the target dynamics (17). 


\section{A. Total Energy Shaping}

For the reasons reported in [25], when the plant contains some inherent dissipation as (12), it is convenient to firstly shape the energy disregarding the inherent dissipation and then to tune the dissipation by damping injection.

Thus, in order to shape the energy of the plant, we consider the following undamped plant, where also the external wrench is disregarded

$$
\left(\begin{array}{c}
\dot{q} \\
\dot{p}
\end{array}\right)=\left(\begin{array}{cc}
0 & I \\
-I & 0
\end{array}\right)\left(\begin{array}{c}
\frac{\partial H}{\partial q} \\
\frac{\partial H}{\partial p}
\end{array}\right)+\left(\begin{array}{c}
0 \\
G
\end{array}\right) u_{e s}
$$

and we design the input $u_{e s}$ in order to obtain an undamped controlled system with the desired energy function $H_{d}$ and with the desired momentum $\bar{p}$.

$$
\left(\begin{array}{c}
\dot{q} \\
\dot{\bar{p}}
\end{array}\right)=\left(\begin{array}{cc}
0 & I \\
-I & 0
\end{array}\right)\left(\begin{array}{c}
\frac{\partial H_{d}}{\partial q} \\
\frac{\partial H_{d}}{\partial \bar{p}}
\end{array}\right)
$$

Since $\bar{p}=M_{d} \dot{q}=M_{d} M^{-1} M \dot{q}=M_{d} M^{-1} p$, we have that the relation between the state of (20) and the state of the target dynamics (21) is given by:

$$
x=\left(\begin{array}{c}
q \\
p
\end{array}\right)=\left(\begin{array}{cc}
I & 0 \\
0 & M M_{d}^{-1}
\end{array}\right)\left(\begin{array}{l}
q \\
\bar{p}
\end{array}\right)=F\left(\begin{array}{l}
q \\
\bar{p}
\end{array}\right)=\Phi(\bar{x}),
$$

and, consequently,

$$
\frac{\partial \Phi}{\partial \bar{x}}=F, \quad \frac{\partial \Phi}{\partial t}=0
$$

Substituting (20), (21), and (23) in (5) we obtain the following matching equations:

$$
\left\{\begin{array}{l}
\frac{\partial H_{d}}{\partial \bar{p}}-\frac{\partial H}{\partial p}=0 \\
G^{\perp}\left\{\frac{\partial H}{\partial q}-M M_{d}^{-1} \frac{\partial H_{d}}{\partial q}\right\}=0 .
\end{array}\right.
$$

It easy to check that the first equation is always satisfied. Furthermore, since both $M$ and $M_{d}$ are constant, using (13) and (19) the second condition can be rewritten as:

$$
G^{\perp}\left\{\frac{\partial V}{\partial q}-M M_{d}^{-1} \frac{\partial V_{d}}{\partial q}\right\}=0
$$

Thus, it is possible to choose $m_{d}$ and $N$ in (18) arbitrarily while the desired potential energy for the controlled system must satisfy (25).

A possible choice for the full rank left annihilator $G^{\perp}$ is

$$
G^{\perp}=\left(\begin{array}{cccccc}
0 & -1 & \frac{G_{1}(2)}{G_{1}(3)} & 0 & 0 & 0 \\
-1 & 0 & \frac{G_{1}(1)}{G_{1}(3)} & 0 & 0 & 0
\end{array}\right)
$$

where $G_{1}(i)$ indicates the $i$-th component of the vector $G_{1}$. Using (26) with (25) yields:

$$
\left\{\begin{array}{l}
\frac{\partial V_{d}}{\partial q_{2}}-\frac{G_{1}(2)}{G_{1}(3)}\left(\frac{\partial V}{\partial q_{3}}-\frac{m}{m_{d}} \frac{\partial V_{d}}{\partial q_{3}}\right)=0 \\
\frac{\partial V_{d}}{\partial q_{1}}-\frac{G_{1}(1)}{G_{1}(3)}\left(\frac{\partial V}{\partial q_{3}}-\frac{m}{m_{d}} \frac{\partial V_{d}}{\partial q_{3}}\right)=0
\end{array}\right.
$$

Admissible potentials are all and only the solutions of the PDEs (27). A possible simple solution is:

$$
V_{d}(q)=-m_{d} g q_{3}+\bar{V}_{d}\left(q_{4}, q_{5}, q_{6}\right)
$$

This potential energy function is consistent with the desired mass $m_{d}$ since it scales the gravity force accordingly and it allows to arbitrarily shape the potential energy of the rotational part.

Remark 2: The non constant terms of (26), and consequently (27) have a singularity corresponding to the configurations where the pitch or the roll are at $\frac{\pi}{2}+k \pi$, where $k \in \mathbb{Z}$. In order for the controller to work properly, the quadrotor should be kept away from these configurations.

Remark 3: The limits in the choice of the potential are due to the underactuation of the quadrotor. Since the attitude is fully actuated, it is possible to arbitrarily choose a potential on the orientation while the underactuation in the Cartesian coordinates limits the choice of a translational potential.

Thus, once an admissible potential has been chosen, using (4), the control input shaping the dynamics of (20) in (21) is given by:

$$
u_{e s}=\left(G^{T} G\right)^{-1} G^{T}\left(\frac{\partial H}{\partial q}-M M_{d}^{-1} \frac{\partial H_{d}}{\partial q}\right)
$$

\section{B. Dissipation and External Wrench Shaping}

We will now consider the full model of the plant and we will design the input $u=u_{e s}+v$ for shaping the damping and the external wrenches.

Considering (22) it is possible to rewrite (12) as:

$$
\begin{gathered}
\left(\begin{array}{c}
\dot{q} \\
\bar{p}
\end{array}\right)=F^{-1}\left(\begin{array}{cc}
0 & I \\
-I & 0
\end{array}\right)\left(\begin{array}{c}
\frac{\partial H}{\partial q} \\
\frac{\partial H}{\partial p}
\end{array}\right)+F^{-1}\left(\begin{array}{l}
0 \\
G
\end{array}\right) u_{e s}- \\
-F^{-1}\left(\begin{array}{cc}
0 & 0 \\
0 & \mathcal{R}
\end{array}\right)\left(\begin{array}{c}
\frac{\partial H}{\partial q} \\
\frac{\partial H}{\partial p}
\end{array}\right)+F^{-1}\left(\begin{array}{l}
0 \\
G
\end{array}\right) v+F^{-1}\left(\begin{array}{l}
0 \\
I
\end{array}\right) w_{e}
\end{gathered}
$$

Considering the results of Sec. IV-A and recalling that

$$
\frac{\partial H}{\partial p}=\frac{\partial H_{d}}{\partial \bar{p}}
$$

we can rewrite (30) as:

$$
\begin{aligned}
\left(\begin{array}{l}
\dot{q} \\
\bar{p}
\end{array}\right)=\left(\begin{array}{cc}
0 & I \\
-I & 0
\end{array}\right) & \left(\begin{array}{c}
\frac{\partial H_{d}}{\partial q_{d}} \\
\frac{\partial H_{d}}{\partial \bar{p}}
\end{array}\right)-\left(\begin{array}{cc}
0 & 0 \\
0 & M_{d} M^{-1} \mathcal{R}
\end{array}\right)\left(\begin{array}{c}
\frac{\partial H}{\partial g_{d}} \\
\frac{\partial H_{d}}{\partial \bar{p}}
\end{array}\right)+ \\
& +\left(\begin{array}{c}
0 \\
M_{d} M^{-1} G
\end{array}\right) v+\left(\begin{array}{c}
0 \\
M_{d} M^{-1}
\end{array}\right) w_{e}
\end{aligned}
$$

Decompose the input as $v=u_{d i}+v_{1}$ and set

$$
u_{d i}=-K_{v} y_{1}
$$

where

$$
y_{1}=G^{T} M^{-T} M_{d}^{T} \frac{\partial H_{d}}{\partial \bar{p}}
$$

is the natural velocity-like output of (31) and

$$
K_{v}=\left(\begin{array}{cc}
k_{T} & 0 \\
0 & K_{R}
\end{array}\right) \in \mathbb{R}^{4 \times 4}
$$

with $k_{T} \in \mathbb{R}^{+}$and $\mathbb{R}^{3 \times 3} \ni K_{R}>0$. The input $u_{d i}$ can be used for tuning the desired damping. Thus, it is possible to rewrite (31) as:

$$
\begin{aligned}
\left(\begin{array}{c}
\dot{q} \\
\dot{p}
\end{array}\right)=\left[\left(\begin{array}{cc}
0 & I \\
-I & 0
\end{array}\right)-\left(\begin{array}{cc}
0 & 0 \\
0 & \mathcal{R}_{d}
\end{array}\right)\right]\left(\begin{array}{c}
\frac{\partial H_{d}}{\partial q} \\
\frac{\partial H_{d}}{\partial \bar{p}}
\end{array}\right)+ \\
+\left(\begin{array}{c}
0 \\
M_{d} M^{-1} G
\end{array}\right) v_{1}+\left(\begin{array}{c}
0 \\
M_{d} M^{-1}
\end{array}\right) w_{e}
\end{aligned}
$$


where

$$
\mathcal{R}_{d}=M_{d} M^{-1} \mathcal{R}+M_{d} M^{-1} G K_{v} G^{T} M^{-T} M_{d}^{T} .
$$

Because of scaling due to the change of the momentum, (33) is not a standard damping injection and it is necessary to verify that $\mathcal{R}_{d}$ is always positive definite. In general the product of two positive definite matrices is not always positive definite. Nevertheless, by simple computations it can be shown that

$$
\mathcal{R}_{d}=\left(\begin{array}{cc}
\left(\frac{m_{d}}{m}\right)^{2} k_{T} G_{1}^{T} G_{1} & 0 \\
0 & k_{d} N+N K_{R} N
\end{array}\right)
$$

The first matrix on the diagonal is trivially positive definite. The second matrix on the diagonal is positive definite because it is the sum of two positive definite matrices. In fact, $k_{d} \in \mathbb{R}^{+}$and therefore $k_{d} N>0$. Furthermore, since $N$ and $K_{R}$ are positive definite, $N K_{R} N$ is positive definite. ${ }^{2}$ The structure of the desired dissipation matrix in (35) is influenced both by the underactuation of the quadrotor and by the change of momentum. Because of the underactuation, the damping in the Cartesian space is influenced only by the parameter $k_{T}$ and, therefore, it is not possible to set arbitrary damping factors along the three Cartesian directions. On the other hand, it is possible to achieve any desired damping for the rotational dynamics by properly tuning the matrix $K_{R}$. The damping force is an external force and, because of the change of momentum in the target dynamics, the desired inertia affects the achievable damping. Nevertheless, setting

$$
\left\{\begin{array}{l}
k_{T}=\left(\frac{m}{m_{d}}\right)^{2} \bar{k}_{T} \\
K_{R}=N^{-1}\left(\bar{K}_{R}-k_{d} N\right) N^{-1}
\end{array}\right.
$$

it is possible to achieve any desired damping $\bar{k}_{T}>0$ along the actuated Cartesian direction and any rotational damping matrix $\bar{K}_{R}>0$.

The change of momentum for the desired target dynamics introduces a scaling also on the way the external wrench $w_{e}$ influences the evolution of the system. Ideally, the external force should influence the evolution of the controlled system in the same way it does in (12). If the external wrench can be measured, then it is possible to exploit the control input for eliminating the scaling.

In order to obtain the ideal behavior, we can see from (33) that the input $v_{1}$ should be chosen in such a way that:

$$
M_{d} M^{-1} G v_{1}+M_{d} M^{-1} w_{e}=w_{e} .
$$

Hovewer, because of the underactuation of the quadrotor, it is possible to have only a partial compensation that can be achieved setting

$$
v_{1}=G^{+}\left(M M_{d}^{-1}\left(I-M_{d} M^{-1}\right) w_{e}\right)+z
$$

where $G^{+}$is the pseudoinverse of $G$ and the term $z$ is an extra control input. Replacing (38) in (37) and setting $z=0$

\footnotetext{
${ }^{2} \forall z \neq 0$ we have $N z=z^{\prime} \neq 0$ and $z^{T}\left(N K_{R} N\right) z=z^{T} N^{T} K_{R} N z=$ $z^{\prime T} K_{R} z^{\prime}>0$.
}

we obtain that:

$$
\begin{aligned}
M_{d} M^{-1} G G^{+}\left(M M_{d}^{-1}\left(I-M_{d} M^{-1}\right) w_{e}\right)+ & \\
& +M_{d} M^{-1} w_{e}=\tilde{w}_{e}
\end{aligned}
$$

where $\tilde{w}_{e}$ is the best compensation that can be achieved.

Remark 4: By simple computations, it can be seen from (38) that the scaling on the external torques can be perfectly compensated and the approximation remains only on the compensation of the translational part.

Finally, putting together (29), (32) and (38), we obtain that the control input $u$ is given by (see also Fig. 2):

$$
\begin{aligned}
& u=u_{e s}+u_{d i}+v_{1}= \\
&=\left(G^{T} G\right)^{-1} G^{T}\left(\frac{\partial H}{\partial q}-M M_{d}^{-1} \frac{\partial H_{d}}{\partial q}\right)- \\
& \quad-K_{v} G^{T} M^{-T} M_{d}^{T} \frac{\partial H_{d}}{\partial \bar{p}}+ \\
& \quad+G^{+}\left(M M_{d}^{-1}\left(I-M_{d} M^{-1}\right) w_{e}\right)+z .
\end{aligned}
$$

which leads to the closed-loop system

$$
\begin{aligned}
\left(\begin{array}{c}
\dot{q} \\
\dot{p}
\end{array}\right)=\left[\left(\begin{array}{cc}
0 & I \\
-I & 0
\end{array}\right)-\left(\begin{array}{cc}
0 & 0 \\
0 & \mathcal{R}_{d}
\end{array}\right)\right] & \left(\begin{array}{l}
\frac{\partial H_{d}}{\partial \partial_{d}} \\
\frac{\partial H_{d}}{\partial \bar{p}}
\end{array}\right)+\left(\begin{array}{l}
0 \\
I
\end{array}\right) \tilde{w}_{e}+ \\
& +\left(\begin{array}{c}
0 \\
M_{d} M^{-1} G
\end{array}\right) z
\end{aligned}
$$

If we set $z=0$; the desired dynamics in (17) as a new quadrotor with a new inertia, damping and potential structure is achieved. The external input $z$ can be used for controlling such a physically modified quadrotor. In other words, the controller in (40) can be used as an inner control loop for changing the physical characteristics of the quadrotor and the input $z$ can be exploited for building outer loops controlling this new system, taking advantage of its new desired physics.

Proposition 2: The controlled system (41) is cyclopassive with respect to the input-output pair:

$$
\left(\left(\begin{array}{c}
z \\
\tilde{w}_{e}
\end{array}\right),\left(G^{T} M^{-T} M_{d}^{T} \frac{\partial H_{d}}{\partial \bar{p}}\right)\right)
$$

Proof: The proof is analogous to that of Prop. 1.

Remark 5: Even if the the compensation of the external wrench is only partial, the target dynamics that is achieved is still well behaved from a physical point of view and no regenerative effects are present. Furthermore if $V_{d}$ can be chosen to be lower bounded in (e.g., in a desired range of operation), then the achievable target dynamics is passive.

\section{SIMULATIONS AND RESULTS}

In this section we present some simulation results to support the theory of proposed control method in this paper. The parameters for the original system dynamics are chosen as follows; $m=1 \mathrm{~kg}, g=9.81 \mathrm{~m} / \mathrm{s}^{2}$. The dissipation on rotational dynamics, as presented in (10), is set to $k_{d}=1$ based on our experiences [3], [4], [6]. We have assumed the aerodynamic drag as $k_{d r a g}=0.5$ in every direction. For more detail, one can check [26]. We first would like to investigate the behavior of different desired masses in free fall case, where there are no external forces or high-level control inputs, i.e., $w_{e}=z=0$. The desired system parameters are 


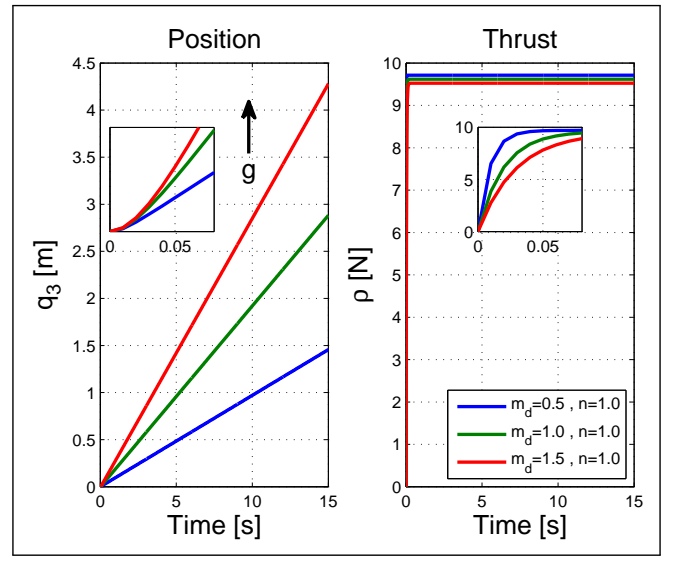

Fig. 3. Free fall for different desired dynamics. The figure on left is the position of the quadrotor along $\vec{z}_{w}$ (height) and the one on the right is the thrust applied by the controller. The small windows show the first 0.08 seconds for both position and thrust, respectively.

chosen as follows: $\bar{k}_{T}=50, \bar{K}_{R}=\operatorname{diag}\left(\bar{k}_{R_{i}}\right)$ for $\bar{k}_{R_{i}}=5$ and $\bar{V}_{d}=-m_{d} g e_{3}+\frac{1}{2} \eta^{T} K_{p} \eta$ where $K_{P}=\operatorname{diag}\left(k_{p_{i}}\right)$ with $k_{p_{i}}=2$. Fig. 3 shows the position $q_{3}$ on the left, and the thrust applied by the controller on the right. The direction of gravity is shown in the plot. It is seen that under the desired viscosity, which is tuned by $k_{T}$, the bigger mass falls faster than the smaller mass. The controller adjusts itself in a way that the quadrotor system behaves as a desired mass.

We also would like to show how we change the rotational dynamics of the system by shaping the desired potential energy. The rotational dynamics of the quadrotor system is fully actuated, hence we have full control on rotational properties. For this, we investigate the impulse response of the rotational dynamics, where the system is hovering. For hovering, we used the high-level control input $z=$ $\left[\begin{array}{llll}m_{d} g & 0 & 0 & 0\end{array}\right]^{T}$ to balance the gravity effect for the desired system. The impulse $1 \mathrm{Nm}$ around $\vec{b}_{b}$ is applied for $1 \mathrm{~s}$. As seen in Fig.4, the system with smaller inertia behaves more compliant to the external torques. The change in orientation $\phi$ reveals that the second order system response, where smaller inertia has bigger magnitude and it requires higher torques to stabilize the system. When we assign a bigger inertia, the system behavior becomes stiffer and rejects the external torques. System reacts instantly and stabilizes itself with less change in orientation. The small inertia comes in handy when for example in safe human-robot-interaction. The big inertia on the other hand might be useful for tasks where the quadrotor needs to reject disturbances quickly, such as maintaining stable contact with a flat surface.

Reshaping the physics, especially rotational dynamics of an underactuated quadrotor system might provide huge advantage for physical interaction of such systems. In order to show this fact, we would like to simulate a sliding on a surface task, where a tool in shape of a rigid stick is connected to the center of gravity (COG) of the quadrotor system, and its tip (tooltip) is in contact with a flat surface. An illustration is shown in Fig.5. This can be interpreted as ceiling painting, cleaning, surface inspection, e.t.c.

In this paper we investigate two cases; first the tooltip is

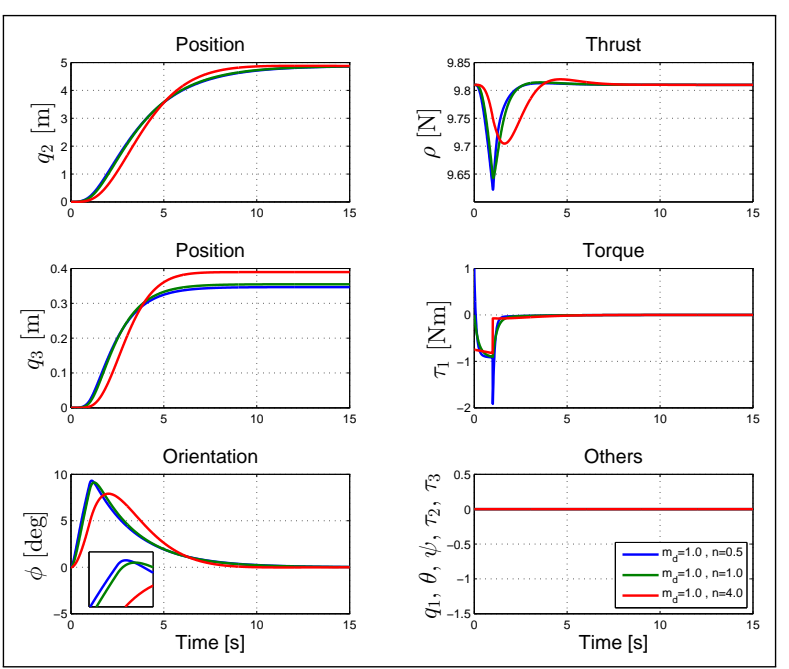

Fig. 4. Impulse response of the rotational dynamics. The external torque is applied around $\vec{b}_{b}$ axis. The small window in orientation is highlighting the change of $\phi$ for different desired inertias. The states and control inputs, which are not effected by the impulse, remained zero.

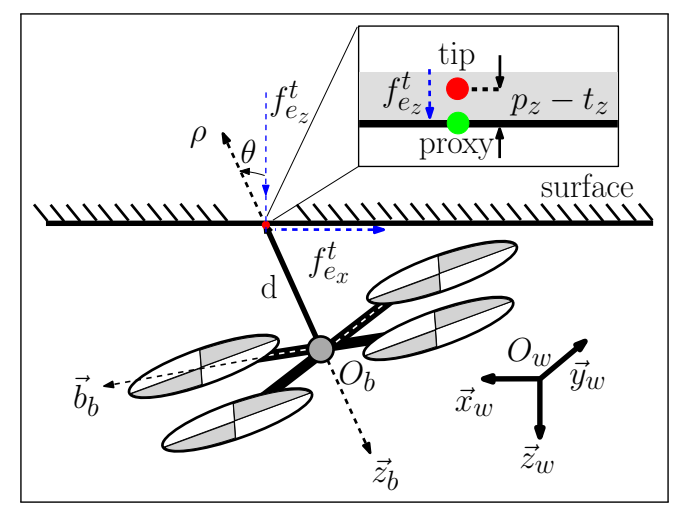

Fig. 5. The tip of a rigid link driven by a quadrotor is in contact with a surface and sliding along one direction. The red point represents the tooltip penetrating the surface (such as ceiling), which is the proxy and presented with green point.

sliding on a flat surface, and second it is sliding on a rough surface, where there are dents and bulges. The surface is placed above (considering $+\vec{z}_{w}$ shows the below) the COG of the quadrotor. We choose to slide along the positive $\vec{x}_{w}$ (See Fig.5). For this, quadrotor needs to be tilted with a certain tilting angle, in this case with $\theta^{*}<0$. A desired attitude can be achieved by shaping the desired potential in a way that it goes to minimum in a desired configuration. Consider the desired rotational potential as

$$
\bar{V}_{d}\left(q_{e}\right)=\frac{1}{2} q_{e}^{T} K_{p} q_{e}
$$

where

$$
q_{e}=\eta-\eta^{*}=\left(\begin{array}{c}
\phi-\phi^{*} \\
\theta-\theta^{*} \\
\psi-\psi^{*}
\end{array}\right)
$$

The desired attitude $\eta^{*}$ is the equilibrium in orientation where the rotational potential goes to minimum. Once the desired attitude is achieved, we need to apply a constant thrust to the system, to maintain the contact with the surface 


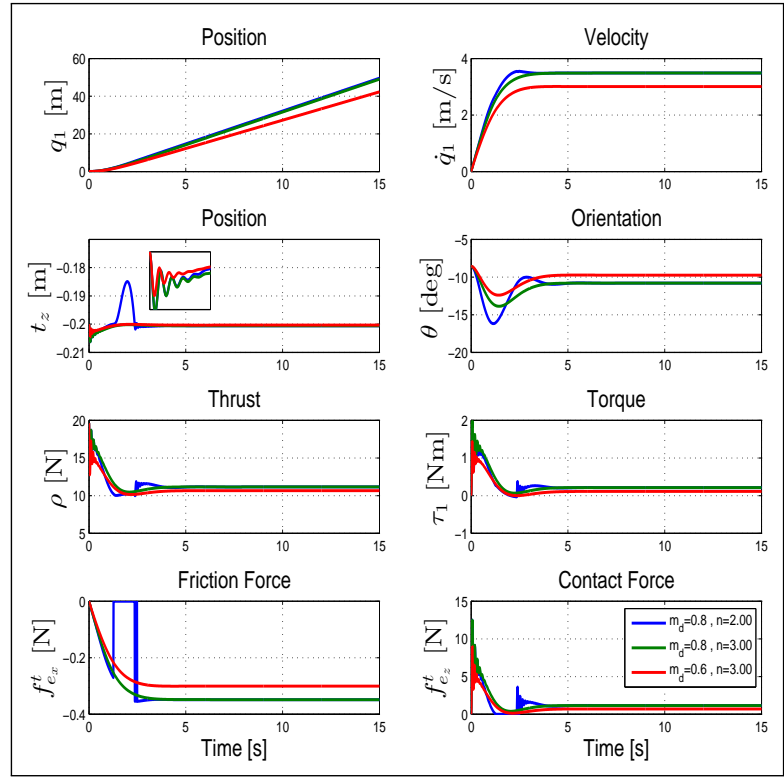

Fig. 6. Stable contact and sliding of tooltip along a flat surface. Small inertia is more vulnerable to the external forces, and smaller mass establishes the contact faster. The hybrid contact modeling is not a problem for passivity based controller in sense of stability.

and to win against the friction forces, so the tooltip can slide along the $+\vec{x}_{w}$ axis. The external forces acting on the tooltip can be considered as: the (contact) reaction force from surface along the $+\vec{z}_{w}$ direction, and friction force against the direction of the sliding motion on the surface. For modeling the reaction of the surface, we used proxy model conceptually introduced in [27], only along the $+\vec{z}_{w}$ direction. In our case, the position of the tooltip is the real position, and the height of the contact surface represents the proxy (See Fig.5). The reaction force from the surface is calculated as

$$
f_{e_{z}}^{t}=k_{\mathrm{wall}}\left(p_{z}-t_{z}\right)
$$

where $k_{\text {wall }}$ is spring gain depending on the characteristics of the surface, $p_{z}$ is the proxy (or surface) position, and $t_{z}$ is the tooltip position along the $\vec{z}_{w}$ axis. For the surface friction, we used a simple viscous friction model [28] such as

$$
f_{e_{x}}^{t}=-\mu \dot{q}_{1}
$$

where $\mu$ is the coefficient of friction, depending on the tooltip and surface characteristics, and $\dot{q}_{1}$ is the velocity of the tooltip (and quadrotor) along $+x_{w}$. In our simulation, we consider a hybrid contact model, where if tooltip penetrates to the surface, then both reaction and friction forces are acting, otherwise there are no external forces, i.e.,

$$
\begin{cases}f_{e}^{t}=0 \in \mathbb{R}^{3}, & \text { if } t_{z}>p_{z} \\
f_{e}^{t}=\left[\begin{array}{lll}
f_{e_{x}}^{t} & 0 f_{e_{z}}^{t}
\end{array}\right]^{T}, & \text { if } t_{z} \leq p_{z}\end{cases}
$$

To calculate the external wrench acting on the COG of the quadrotor, we use the following transformation

$$
w_{e}=\left(\begin{array}{cc}
I & 0 \\
S(d) R^{T} & R^{T}
\end{array}\right) w_{e}^{t}
$$

where $d$ is the distance between COG of the quadrotor and the tooltip, $w_{e}^{t}=\left[f_{e}^{t^{T}} \tau_{e}^{t^{T}}\right]^{T}$ is the external wrench acting on the tooltip. In our case, $\tau_{e}^{t}=0$. For the wall characteristics, we assigned $k_{\text {wall }}=2000$, and $\mu=0.1$. To win the friction force and start sliding, it is necessary that the angle between normal of the surface and the applied force must satisfy

$$
\left|\theta^{*}\right|>\tan ^{-1}(\mu)
$$

hence, we choose $\theta^{*}=-0.15 \mathrm{rad} \simeq-8.6 \mathrm{deg}$. A constant thrust of $2 m g \mathrm{~N}$ is applied using $z$ to maintain the contact and to slide along $+x_{w}$. The distance between tooltip and COG of the quadrotor is chosen as $d=\left[\begin{array}{lll}0.2 & 0 & -0.2\end{array}\right]^{T}$, in units of meters, for the reason explained in [16]. For desired rotational potential, we set $k_{p_{i}}=5.5$. The desired damping along the thrust direction is set to $\bar{k}_{T}=10$ and for the rotational dynamics it is $\bar{k}_{R_{i}}=50$. The proxy position is set to $p_{z}=-0.2 \mathrm{~m}$. Fig. 6 shows the results for different desired mass and inertia values. By judging the change of $t_{z}$, and orientation $\theta$, bigger inertia quickly adapts to the disturbances, while smaller inertia is oscillating, which causes disconnection with the contact surface (blue plot). It is noticed that a smaller mass (red plot) establishes the contact with the surface faster than the bigger mass. This shows how the quadrotor can benefit from the proposed controller, where we shape and dissipate both kinetic and potential energies. Changing the desired mass creates a difference in orientation at steady state, since the total force (with surface reaction) along $\vec{z}_{w}$ creates bigger torque for bigger mass, which is directly related to the length of the tool. This is an important motivation of choosing a reasonable $d$ value, which is a possible topic of study for future works. Note that the maximum penetration of the red plot to the surface is calculated as $4 \mathrm{~mm}$, and the final penetration is $0.3 \mathrm{~mm}$.

As explained before, the external forces are modeled discontinuously. It is seen in the blue plot of Fig. 6, the tooltip loses the contact with the surface, yet the controller stabilizes the system anyway. In fact, an advantage of passivity based controllers is that they stabilize (hybrid) systems, where discontinuities may exist.

In the second case, the quadrotor slides on a rough surface, where there are dents and bulges. For this, we simply change the position of the proxy, $p_{z}$, and let the quadrotor slide on this new surface. Different from the previous simulation, we set $k_{p_{i}}=10$ and $\bar{k}_{T}=15$. The results are shown in Fig. 7. The position of proxy is presented as black dashed plot, where it is first shifted $3 \mathrm{~cm}$ outwards, i.e., representing a bulge, and later $3 \mathrm{~cm}$ inwards, i.e., representing a dent. Notice that the direction of the gravity is also given on the figure. As it is expected from the outcome of previous simulations, system with smaller inertia has more compliant reaction to the surface changes. Again, the discontinuity of the external forces does not cause instabilities, thanks to the passivity based controller. One has to notice that by tuning the parameters such as $K_{P}, \bar{k}_{R}$ and $\bar{k}_{T}$, and setting desired mass and inertia values, it is always possible to change the physical behavior of the system depending on the desired objective.

We encourage the interested reader to watch the video attached to this paper where we visually present the simulations described in this section. 


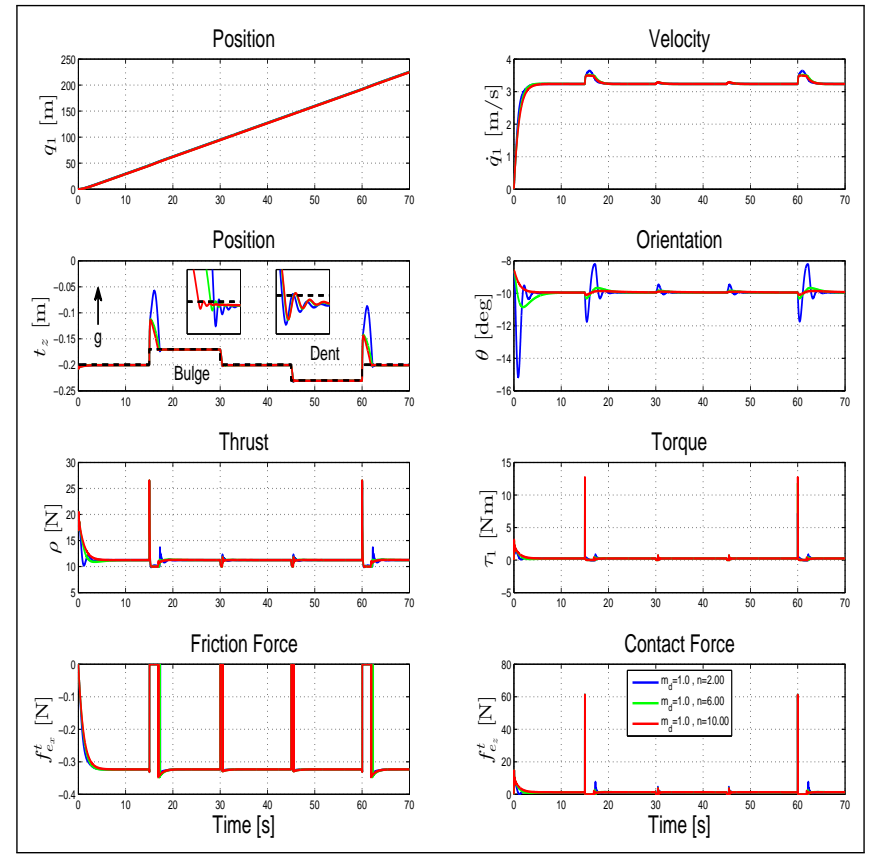

Fig. 7. Stable contact and sliding along a rough surface, where there are dents and bulges. Zoomed windows in the tooltip position plot highlight the behavior of the system when a bulge and a dent are encountered, respectively. Bigger inertia (red plot) reestablishes the contact with the surface faster, while smaller inertia (blue and green plots) are more vulnerable to the external forces.

\section{CONCLUSIONS}

In this work we have illustrated how IDA-PBC and energy based control techniques can be exploited for controlling the interactive behavior of an underactuated quadrotor. We showed how to change the physics of such a system by shaping its total energy, by setting a desired damping and by scaling external wrenches for achieving a desired dynamics. Simulations have shown the effectiveness of the controller considering two sliding tasks, that can be interpreted as a ceiling painting, cleaning or surface inspection by a quadrotor. Future work aims at experimental validation of the controller developed in this work. Furthermore, we want to extend the strategy outlined in the paper to the case where one or more manipulators are mounted on a flying base.

\section{REFERENCES}

[1] V. Mistler, A. Benallegue, and N. K. M'Sirdi, "Exact linearization and noninteracting control of a 4 rotors helicopter via dynamic feedback," in 10th IEEE Int. Symp. on Robots and Human Interactive Communications, Bordeaux, Paris, France, Sep. 2001, pp. 586-593.

[2] T. Lee, M. Leokyand, and N. H. McClamroch, "Geometric tracking control of a quadrotor UAV on SE(3)," in 49th IEEE Conf. on Decision and Control, Atlanta, GA, Dec. 2010, pp. 5420-5425.

[3] D. J. Lee, A. Franchi, H. I. Son, H. H. Bülthoff, and P. Robuffo Giordano, "Semi-autonomous haptic teleoperation control architecture of multiple unmanned aerial vehicles," IEEE/ASME Trans. on Mechatronics, Focused Section on Aerospace Mechatronics, vol. 18, no. 4, pp. 1334-1345, 2013.

[4] A. Franchi, C. Secchi, M. Ryll, H. H. Bülthoff, and P. Robuffo Giordano, "Shared control: Balancing autonomy and human assistance with a group of quadrotor UAVs," IEEE Robotics \& Automation Magazine, Special Issue on Aerial Robotics and the Quadrotor Platform, vol. 19, no. 3, pp. 57-68, 2012.

[5] O. Bourquardez, R. Mahony, N. Guenard, F. Chaumette, T. Hamel, and L. Eck, "Image-based visual servo control of the translation kinematics of a quadrotor aerial vehicle," IEEE Trans. on Robotics, vol. 25, no. 3 , pp. 1552-3098, 2009.
[6] A. Franchi, C. Masone, V. Grabe, M. Ryll, H. H. Bülthoff, and P. Robuffo Giordano, "Modeling and control of UAV bearingformations with bilateral high-level steering," The International Journal of Robotics Research, Special Issue on 3D Exploration, Mapping, and Surveillance, vol. 31, no. 12, pp. 1504-1525, 2012.

[7] P. Robuffo Giordano, A. Franchi, C. Secchi, and H. H. Bülthoff, "A passivity-based decentralized strategy for generalized connectivity maintenance," The International Journal of Robotics Research, vol. 32, no. 3, pp. 299-323, 2013.

[8] AIRobots, "EU Collaborative Project ICT-248669," www.airobots.eu.

[9] ARCAS, "EU Collaborative Project ICT-287617," www.arcas-project. eu.

[10] F. Augugliaro and R. DAndrea, "Admittance control for physical human-quadrocopter interaction," in European Control Conference, Zurich, Switzerland, July 2013, pp. 1805-1810.

[11] S. Bellens, J. De Schutter, and H. Bruyninckx, "A hybrid pose/wrench control framework for quadrotor helicopters," in 2012 IEEE Int. Conf. on Robotics and Automation, St.Paul, MN, May 2012, pp. 2269-2274.

[12] K. Sreenath and V. Kumar, "Dynamics, control and planning for cooperative manipulation of payloads suspended by cables from multiple quadrotor robots," in Robotics: Science and Systems, Berlin, Germany, June 2013.

[13] V. Lippiello and F. Ruggiero, "Exploiting redundancy in cartesian impedance control of UAVs equipped with a robotic arm," in 2012 IEEE/RSJ Int. Conf. on Intelligent Robots and Systems, Vilamoura, Portugal, Oct. 2012, pp. 3768-3773.

[14] M. Fumagalli, R. Naldi, A. Macchelli, R. Carloni, S. Stramigioli, and L. Marconi, "Modeling and control of a flying robot for contact inspection," in 2012 IEEE/RSJ Int. Conf. on Intelligent Robots and Systems, Vilamoura, Portugal, Oct 2012, pp. 3532-3537.

[15] M. Orsag, C. Korpela, and P. Oh, "Modeling and control of MM-UAV: Mobile manipulating unmanned aerial vehicle," Journal of Intelligent \& Robotics Systems, vol. 69, no. 1-4, pp. 227-240, 2013.

[16] D. J. Lee and C. Ha, "Mechanics and control of quadrotors for tool operation," in 2012 ASME Dynamic Systems and Control Conference, Fort Lauderdale, FL, Oct. 2012.

[17] G. Gioioso, M. Ryll, D. Prattichizzo, H. H. Bülthoff, and A. Franchi, "Turning a near-hovering controlled quadrotor into a 3D force effector," in 2014 IEEE Int. Conf. on Robotics and Automation, Hong Kong, China, May. 2014.

[18] P. E. Pounds and A. M. Dollar, "UAV rotorcraft in compliant contact: Stability analysis and simulation," in 2011 IEEE/RSJ Int. Conf. on Intelligent Robots and Systems, San Francisco, CA, September 2011, pp. 2660-2667.

[19] A. Y. Mersha, R. Carloni, and S. Stramigioli, "Port-based modeling and control of underactuated aerial vehicles," in 2011 IEEE Int. Conf. on Robotics and Automation, Shanghai, China, May 2011, pp. 14-19.

[20] R. Ortega, A. van der Scahft, B. Maschke, and G. Escobar, "Interconnection and damping assignment passivity-based control of portcontrolled hamiltonian systems," Automatica, vol. 38, no. 4, pp. 585596, 2002.

[21] Z. Wang, P. Goldsmith, and J. Gu, "Regulation control of underactuated mechanical systems based on a new matching equation of portcontrolled hamiltonian systems," in 2009 IEEE Int. Conf. on Robotics and Automation, Kobe, Japan, May 2009, pp. 992-997.

[22] C. Secchi, S. Stramigioli, and C. Fantuzzi, Control of Interactive Robotic Interfaces: a port-Hamiltonian Approach, ser. Tracts in Advanced Robotics. Springer, 2007.

[23] M. Cutler, N. K. Ure, B. Michini, and J. P. How, "Comparison of fixed and variable pitch actuators for agile quadrotors," in AIAA Conf. on Guidance, Navigation and Control, Portland, OR, Aug. 2011.

[24] J. C. Willems, "Dissipative dynamical systems part I: General theory," Archive for Rational Mechanics and Analysis, vol. 45, no. 5, pp. 321$351,1972$.

[25] F. Gomez-Estern and A. J. van der Schaft, "Physical damping in IDAPBC controlled underactuated mechanical systems," European Journal of Control, vol. 10, no. 5, pp. 451-468, 2004.

[26] J. B. Brandt and M. S. Selig, "Propeller performance data at low reynolds numbers," in AIAA Aerospace Sciences Meeting, Orlando, FL, Jan. 2011.

[27] C. B. Zilles and J. K. Salisbury, "A constraint-based god-object method for haptic display," 1995 IEEE/RSJ Int. Conf. on Intelligent Robots and Systems, vol. 3, no. Pittsburgh, PA, pp. 146-151, 1995.

[28] H. Olsson, K. J. Astrom, M. Gafvert, C. C. D. Wit, and P. Lischinsky, "Friction models and friction compensation," European Journal of Control, vol. 4, no. 3, pp. 176-195, 1998. 